\title{
An Unusual Case of Drug-Induced Linear IgA Bullous Disease
}

By Robert Bobotsis HBSc, Michael Sawchuk MD FRCPC, Jenny Shu MD, FRCPC, Mariamma G. Joseph MD FRCPC

\author{
About the Authors: \\ Robert Bobotsis is with the Schulich School of Medicine and Dentistry and Western University, London, Ontario, Canada. \\ Michael Sawchuck is with the Department of Dermatology, University of Ottawa, Ottawa ON. \\ Mariamma G. Joseph is with the Department of Pathology, London Health Sciences Centre and Western University, London, Ontario, \\ Canada. \\ Jenny Shu is with the Division of Rheumatology, University of Toronto, Toronto ON.
}

\begin{abstract}
We describe a case of vancomycin-induced linear IgA bullous dermatosis (LABD) with unusual features in a 75-year old woman who received intravenous vancomycin for methicillin-resistant Staphylococcus Aureus infection. Our patient demonstrated an unusual lesional distribution with initial facial involvement and a longer latent period than usual, which to our knowledge has not yet been reported in the literature. A skin punch biopsy including direct immunofluorescence study (IMF) confirmed the diagnosis of LABD. The patient's cutaneous eruptions resolved over the next 14 days with stoppage of medication and supportive care without the need for systemic steroids. Physicians should be aware of this rare type of autoantibody mediated drug reaction in patients put on vancomycin. Early diagnosis using a skin punch biopsy with direct IMF study would avoid unnecessary laboratory investigations and initiate prompt management.

\section{Résumé}

Nous décrivons ici un cas de dermatose bulleuse à IgA linéaire (DIgAL) d’origine médicamenteuse et aux caractéristiques inhabituelles, chez une femme âgée de 75 ans ayant reçu de la vancomycine par voie intraveineuse pour traiter une infection causée par un Staphylococcus Aureus résistant à la méthicilline. La patiente présentait une distribution inhabituelle des lésions avec une atteinte initiale à la face et une période de latence plus longue que la normale. À notre connaissance, cela n’a jamais été rapporté dans la littérature. Une biopsie à l'emporte-pièce de la peau, doublée d'un test d'immunofluorescence directe, a confirmé le diagnostic de DIgAL. Les lésions cutanées de la patiente se sont résorbées dans les quatorze jours suivant l’arrêt du médicament, avec des soins de soutien et sans recours à des stéroïdes systémiques. Il est important que les médecins soient informés de ce type rare de réaction médicamenteuse à médiation d'autoanticorps chez les patients sous vancomycine. Un diagnostic précoce par biopsie à l'emporte-pièce et test d'immunofluorescence directe permet d'éviter des examens de laboratoire superflus et de débuter une prise en charge rapide.
\end{abstract}




\section{Case Synopsis}

A 75-year-old woman presented to the emergency department with an altered level of consciousness and sepsis requiring intubation. She had been treated with Keflex for a wound infection post L5S1 lumbar discectomy and fusion surgery, but now presented with an epidural abscess and ventriculitis confirmed to be due to methicillin-resistant Staphylococcus Aureus bacteremia. The patient was taking naproxen, baclofen, levothyroxine, fenofibrate, omeprazole, sotalol, hydrochlorothiazide, ativan, zopiclone, and acetaminophen. The patient was started on vancomycin just prior to duraplasty with incision and drainage. 27 days

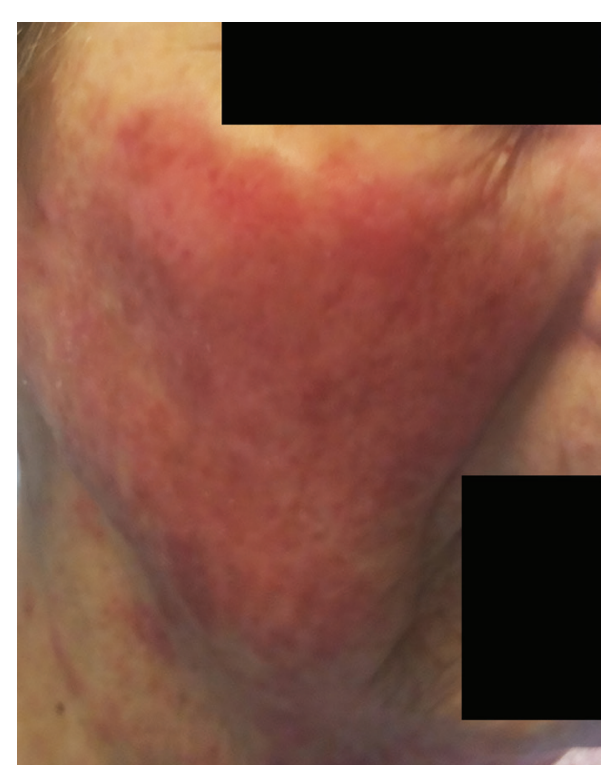

A

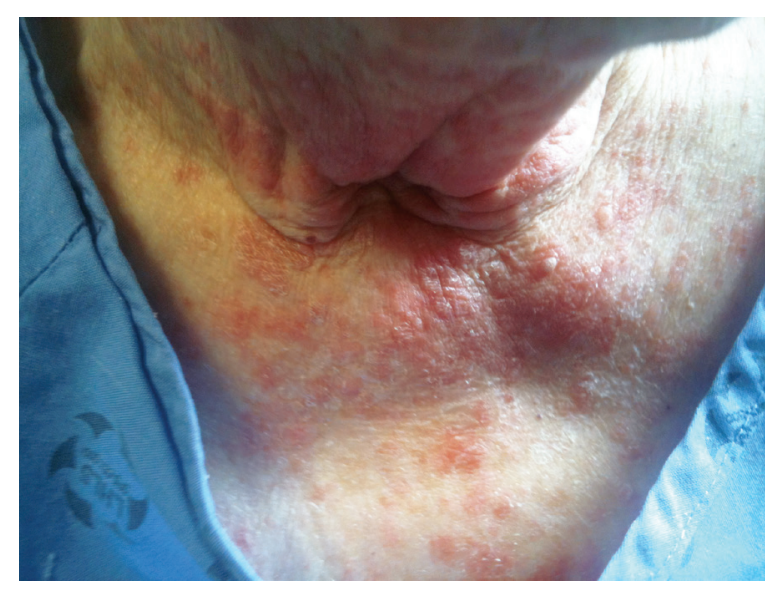

C later, she developed a morbilliform maculopapular cutaneous eruption, which started on her face before progressing diffusely to the trunk and limbs (Figure 1A). The lesions were pruritic and urticarial with blanchable, erythematous, well-demarcated plaques containing some fine scale (Figure 1B). Blistering began ten days after presentation of the eruption resulting in erosion (Figure 1C and Figure 1D). There was no mucous membrane involvement or fever present.

Investigations were unremarkable except for an elevated eosinophil level of $2.6 \times 10^{9} / \mathrm{L}$ and mild leukocytosis of 11.2 $\times 10^{9} / \mathrm{L}$. Peak serum vancomycin was found to be within
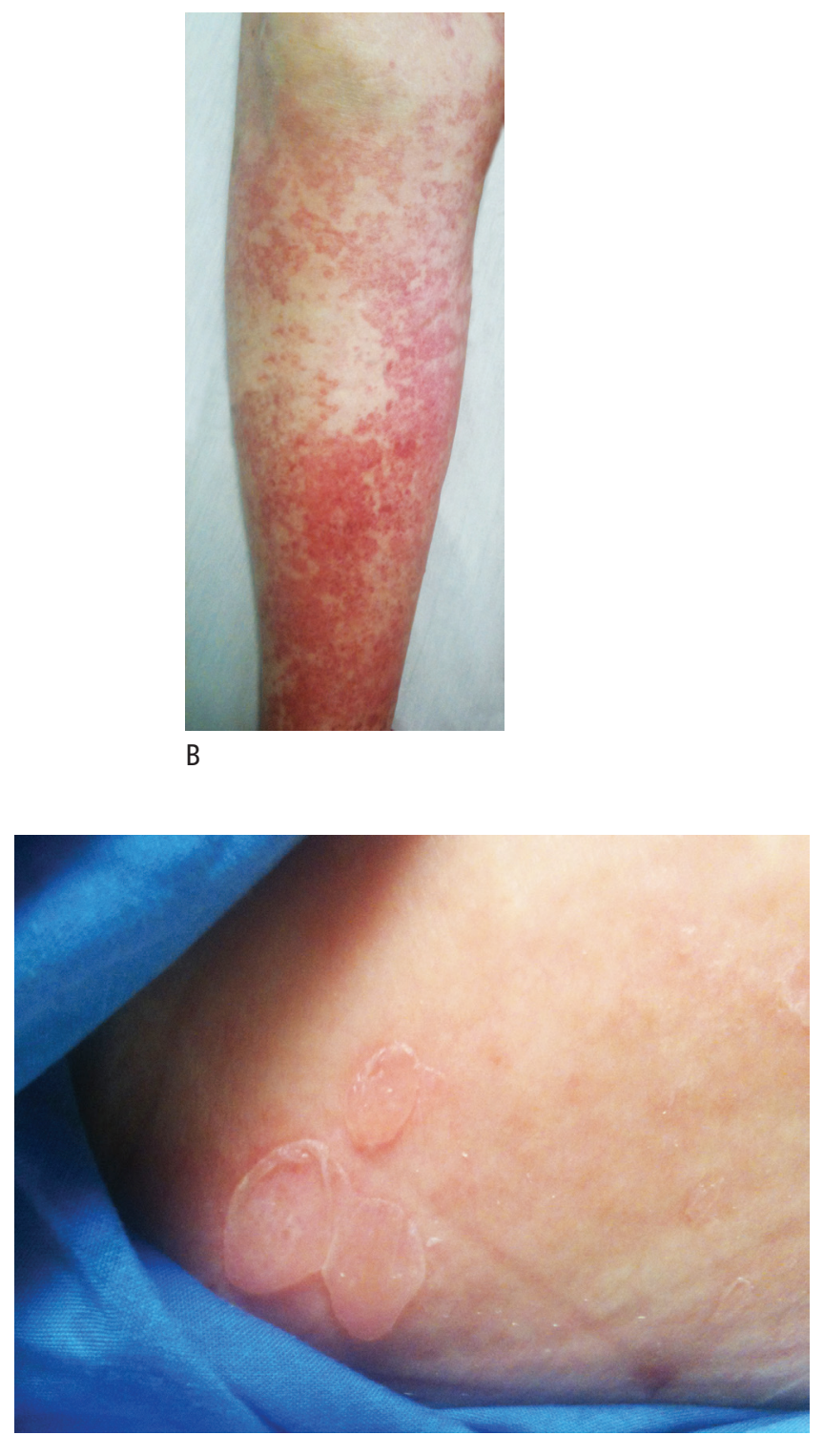

Figure 1. (A) Initial presentation of malar erythema on face. (B) Eruption involving extremities with a maculopapular (morbilliform) appearance. (C\&D) Blistering began ten days after initial presentation of eruption. 
therapeutic range at $21.2 \mu \mathrm{mol} / \mathrm{L}$. A drug rash with eosinophilia and systemic symptoms (DRESS) syndrome was originally suspected; however, the patient had no fever nor any systemic symptoms making this diagnosis unlikely. A punch biopsy of skin lesion was performed for definitive diagnosis. Vancomycin was discontinued and changed to linezolid and the patient received intravenous fluids and topical steroids (betamethasone valerate $0.05 \%$ with $0.25 \%$ camphor and $0.25 \%$ menthol to affected areas of the body and valerate $2 \%$ to lesions on the face) to treat the eruption. The patient's cutaneous eruptions resolved over the next 14 days with additional supportive care, which included pain control, fluid and electrolyte management and nutritional support without the need for systemic steroids.

Histologic examination of the diagnostic skin punch biopsy showed a subepidermal blister with abundant inflammatory infiltrate in the dermis composed of numerous neutrophils, a few eosinophils and lymphocytes. The blister contained fibrin, neutrophils and eosinophils (Figure 2A and Figure 2B). Punch biopsy of peri-lesional skin for direct immunofluorescence (DIF) study showed IgA deposition in a linear fashion along the basement membrane zone (Figure 2C) confirming the diagnosis of LABD.

\section{Discussion}

Linear immunoglobulin A ( $\operatorname{IgA}$ ) bullous dermatosis (LABD) is an acquired autoimmune subepidermal blistering disease of the skin and/or mucous membranes, which is defined by the histopathological finding of subepidermal vesicles with predominantly neutrophilic infiltration and linear IgA deposits in the basement membrane zone. This disease can occur in

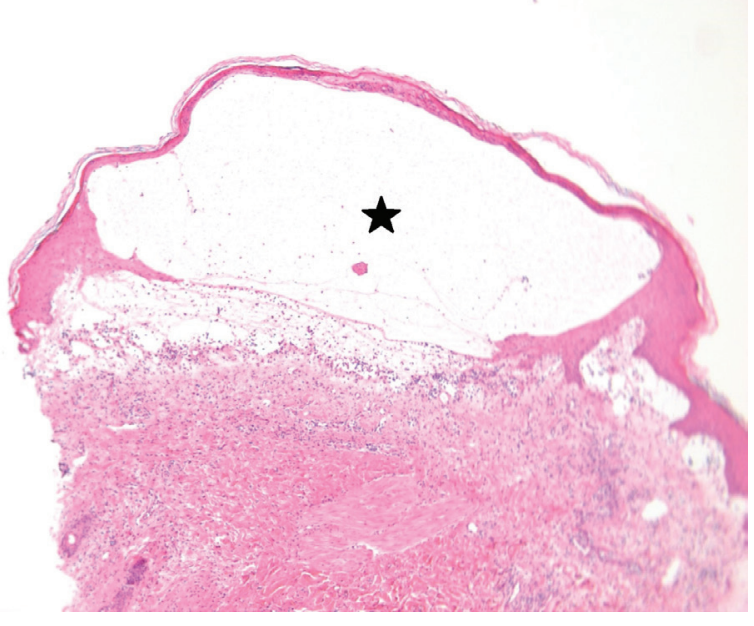

A

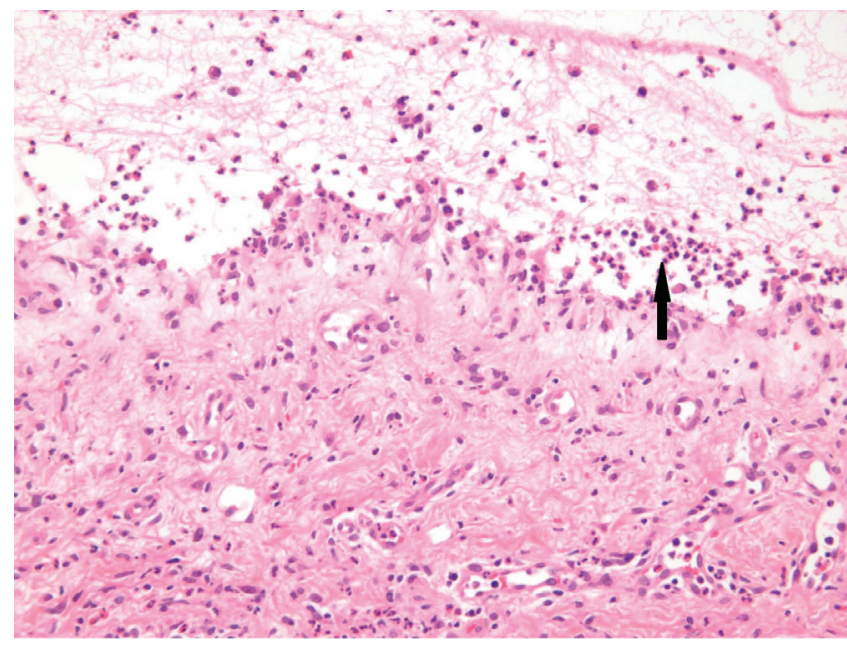

B

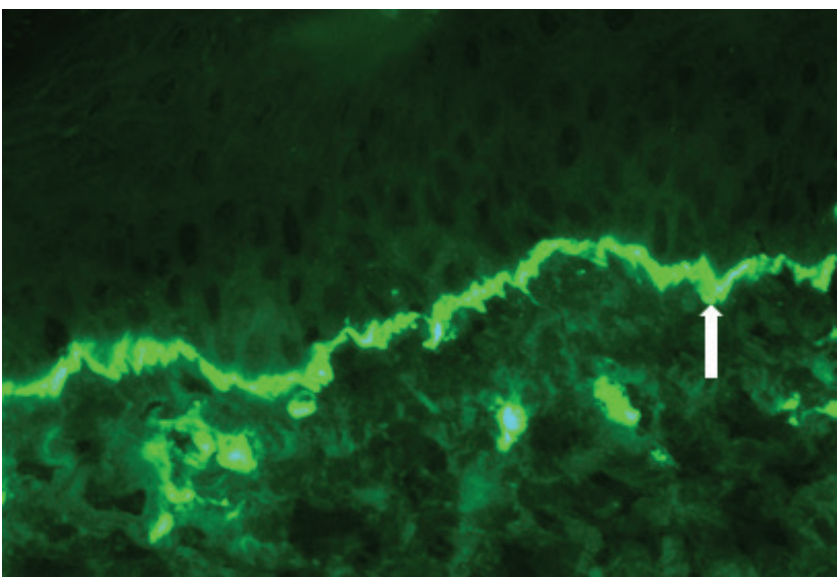

Figure 2. (A) Hematoxylin and eosin (H \& E) stain of punch biopsy skin back shows a subepidermal blister (star) (B) Higher magnification showing abundant neutrophils (arrow, H\&E stain). (C) Direct immunofluorescence (IMF) test on the perilesional skin shows linear IgA deposition along the basement membrane zone (arrow). 
idiopathic and drug-induced forms. While the idiopathic form is more often seen in children and has been associated with specific HLA haplotypes, drug-induced LABD occurs predominantly in adulthood. ${ }^{1,2}$ Drug-induced LABD has been reported with many drugs, including amiodarone, ampicillin, captopril, cefamandole, cyclosporine, diclofenac, glibenclamide, INF-gamma, IL-2, lithium, penicillin $\mathrm{G}$, phenytoin, piroxicam, somatostatin, trimethoprim, vigabatrin, NSAIDs and acetaminophen. ${ }^{3,4}$ However, it has been most consistently associated with intravenous administration of vancomycin, which is responsible for approximately half the cases in the literature, ${ }^{5}$ and does not appear to be dose dependent. ${ }^{3}$ Although our patient was taking other medications that can precipitate LABD (NSAIDs and acetaminophen), the onset and time course of the disease strongly suggest that vancomycin is the causative agent.

Vancomycin is known to produce a diverse variety of adverse drug reactions including the red man syndrome, which is an infusion-related reaction peculiar to vancomycin. Non- bullous maculopapular rash is the most frequent type of skin eruption, a hypersensitivity reaction. Systemic involvement causing DRESS syndrome has been rarely reported. Severe drug reactions such as leukocytoclastic vasculitis, extensive fixed drug eruptions (which are localized), Stevens-Johnson syndrome, toxic epidermal necrolysis and LABD are very rare.

Vancomycin-induced LABD is a subepidermal blistering eruption, which is classically characterized by the appearance of many pruritic tense bullae on a base of annular erythema. However, the presentation can clinically mimic a variety of other vesiculobullous diseases, including patterns resembling dermatitis herpetiformis, bullous pemphigoid, erythema multiforme, and even more severe disorders like Stevens-Johnson syndrome and toxic epidermal necrolysis which makes the diagnosis difficult. ${ }^{6}$ The histologic differential diagnoses for subepidermal blistering disease include bullous pemphigoid and dermatitis herpetiformis. Although there is histologic overlap between these conditions, predominance of neutrophils in the infiltrate and the classic IMF pattern of linear deposition of IgA along the basement membrane zone confirmed the diagnosis of LABD in our patient.

Our patient presented with some unique clinical features worth mentioning. Firstly, cases of vancomycin-induced LABD in the literature are varied, but lesions have been reported to appear within 1-3 weeks of vancomycin administration. ${ }^{1,3,7}$ This is in contrast to the latency period of this case, where our patient's lesions erupted almost one month after vancomycin administration. Additionally, cases in literature have most commonly reported cutaneous involvement affecting the limbs and trunk with sparing usually of the face and neck. ${ }^{1,89}$ However, our patient presented with a malar type eruption on the face at the initial presentation before spreading to the extremities.
The variability in the presentation (latency period, lesional morphology, distribution) is important for clinicians to recognize so vancomycin-induced LABD is kept in the differential diagnosis and confirmatory skin biopsies are performed without delay. The variability of vancomycin-induced LABD may reflect the pathophysiology of the disease, which has still not been fully elucidated. Like other drug-induced immune conditions, it is possible the vancomycin cross-reacts with a component of the epidermal basement membrane, exposes sequestered antigens or alters the confirmation of epitopes resulting in the formation of autoantibodies. ${ }^{10}$ While the target antigens in idiopathic LABD have been extensively investigated, there are few reports of the causative target antigens in the drug-induced form. ${ }^{2}$ Reported basement membrane zone (BMZ) antigens include a $97 \mathrm{KDa}$ protein (which may be identical to the extracellular portion of BPAg2), a 230 KDa protein (similar to BPAg1), BP180, LAD285, unidentified $210 \mathrm{KDa}, 130 \mathrm{KDa}$ and $83 \mathrm{KDa}$ antigens. ${ }^{2,4,5,7,11}$ Most recently, IgA antibodies to the $145-$ and $165-\mathrm{kDa}$ a 3 subunits of laminin-332 have been reported in a case of vancomycin-induced LABD. ${ }^{12}$ The fact IgA autoantibodies have been reported to react with numerous $\mathrm{BMZ}$ antigens may explain why the disease presentation is so heterogeneous. Other laboratory investigations also underscore the variable and unpredictable manifestations of this drug-induced cutaneous disease because in addition to linear IgA deposition along the BMZ, patients may also have deposition of $\mathrm{C} 3$, IgG and circulating IgG and IgA autoantibodies (none of which were seen in our patient). ${ }^{8,9}$

A careful drug history is extremely important as immunohistopathological and clinical features are identical between drug-induced and idiopathic forms, yet prognosis and treatment are quite different. ${ }^{1}$ The prognosis of drug-induced LABD is usually favourable as patients experience remission with drug withdrawal, while spontaneous remission in the idiopathic form is $10-50 \%{ }^{7}$ Idiopathic LABD usually requires dapsone and if needed topical or oral steroid treatment, but its course may be protracted. ${ }^{13}$

In conclusion, we report a case of vancomycin-induced LABD, which presented with a few unique clinical features that to the authors' best knowledge have not been previously reported in the literature. Specifically, our patient's morbilliform eruption started almost one month after the initiation of vancomycin and began on the face before spreading to the extremities. Prompt histological evaluation with IMF study allows precise diagnosis of this condition. This case report highlights the importance of keeping LABD in the differential diagnosis of vancomycininduced blistering drug reactions which would prompt clinicians to perform a diagnostic skin biopsy, make a rapid diagnosis, initiate appropriate treatment and avoid unnecessary delay and investigations. 


\section{Disclosure}

The authors have no conflicts of interest.

Ethics approval is not required for this type of research at our institution.

\section{References}

1. Fortuna G, Salas-Alanis JC, Guidetti E, et al. A critical reappraisal of the current data on drug-induced linear immunoglobulin A bullous dermatosis: A real and separate nosological entity? JAAD 2012;66:988-94.

2. Palmer RA, Ogg G, Allen J, et al. Vancomycin-induced linear IgA disease with autoantibodies to BP180 and LAD 285. Br J Dermatol 2001;145:816-20.

3. Neughebauer BI, Negron G, Pelton S, et al. Bullous skin disease: an unusual allergic reaction to vancomycin. Am J Med Sci 2002;323:273-8.

4. Chanal J, Ingen-Housz-Oro S, Ortonne N, et al. Linear IgA bullous dermatosis: comparison between the drug-induced and spontaneous forms. Br J Dermatol 2013;169:1041-8.

5. Eisendle K, Bonatti H, Sepp N, et al. Vancomycin-induced linear IgA bullous dermatosis in an immunosuppressed transplant recipient. JEADV 2007;21:996-97.
6. Delavalle RP, Burch JM, Tayal S, et al. Vancomycin-associated linear IgA bullous dermatosis mimicking toxic epidermal necrolysis. JAAD 2003;48:S56-7.

7. Waldman MA, Black DR, Callen JP. Vancomycin-induced linear IgA bullous disease presenting as toxic epidermal necrolysis. Clin Exp Dermatol 2004;29:633-6.

8. Armstrong AW, Fazeli A, Yeh SW, et al. Vancomycin-induced linear IgA disease manifesting as bullous erythema multiforme. J Cutan Pathol 2004;31:393-97.

9. Nousari HC, Kimyai-Asadi A, Caeiro JP, et al. Clinical, demographic and immunohistologic features of vancomycin-induced linear IgA bullous disease of the skin. Medicine (Baltimore) 1999;78:1-8.

10. Selvaraj PK, Khasawneh FA. Linear IgA bullous dermatosis: a rare side effect of vancomycin. Ann Saudi Med 2013;33(4):397-99.

11. Paul C, Wolkenstein P, Prost C, et al. Drug-induced linear IgA disease: target antigens are heterogeneous. BJD 1997;136(3):406-11.

12. Zenke $\mathrm{Y}, \mathrm{Nakano} \mathrm{T}$, Eto $\mathrm{H}$, et al. A case of vancomycin-associated linnear IgA bullous dermatosis and IgA antibodies to the $\alpha 3$ subunit of laminin-332. BJD 2013;170(4):965-69.

13. Jones DH, Todd M, Craig TJ. Early diagnosis is key in vancomycin-induced linear IgA bullous dermatosis and Stevens-Johnson syndrome. JAOA 2004;104:157-63. 